\title{
SPECTRAL DECOMPOSITION OF SPHERICAL IMMERSIONS WITH RESPECT TO THE JACOBI OPERATOR
}

\author{
by J. ARROYO, M. BARROS and O. J. GARAY ${ }^{\dagger}$
}

(Received 1 October, 1996; revised 8 January, 1997)

\begin{abstract}
We study the spectral decomposition with respect to the Jacobi operator, $J$, of spherical immersions and characterize those with a simple decomposition in terms of the Finite Chen-type submanifolds. As a consequence, we give an application to the inverse problem for $J$.
\end{abstract}

1. Introduction. The Jacobi operator $J$ (or the second variation operator) was introduced by Simons in [11]. It appears in the study of the second variation formula of the area function for a compact minimal submanifold $M$ of a Riemannian manifold $\tilde{M}$ and it is an elliptic operator acting on the normal bundle of $M, N(M)$. A cross-section $V$ of $N(M)$ is a $J a c o b i$ field if $J V=0,[11]$. This definition is a generalization of the Jacobi fields over geodesics. Spectral properties of this operator have been studied in [7], [9].

For another classical elliptic operator, the Laplacian $\Delta$, the spectral behaviour of an isometric immersion of a Riemannian manifold $M$ in an Euclidean space has been widely studied: finite type submanifolds (a generalization of minimal submanifolds) were introduced by B.-Y. Chen in the late seventies and can be characterized by a variational minimal principle. For a recent survey on this subject, see [6].

We have found in [2] a relation between both elliptic operators (one can see also [10]). This suggests studying the spectral behaviour with respect to $J$ of the position vector of spherical submanifolds and relate it to the Chen-type of the submanifold.

In this paper, we first define the notion of finite J-type for spherical submanifolds. Then, we analyse those spherical submanifolds with the easiest spectral decomposition with respect to $J$. Thus, we find that $J$-type 1 characterizes minimality in the sphere and therefore is equivalent to the Chen-type 1 . The following step is to study $J$-type 2 spherical immersions. We find that, analogously to the previous case, this family coincides with that of Chen-type 2 for hypersurfaces. Finally, we use this to see that there exists a spectral condition for a spherical hypersurface with constant mean and scalar curvatures to be totally geodesic.

2. Preliminaries. Let $M^{p}$ be a Riemannian manifold of dimension $p$, isometrically immersed in a Riemannian manifold $\tilde{M}^{n}$ of dimension $n=p+q$. The normal bundle of $M^{p}, N(M)$ is then a real q-dimensional vector bundle with inner product induced by the metric of $\tilde{M}^{n}$.

Let $J$ be the Jacobi operator (or the second variation operator) acting on cross-sections $\xi$ of $N(M)$. It is a second order differential operator which is defined by

$$
\begin{aligned}
& J: N(M) \rightarrow N(M), \\
& J \xi=\left(\Delta^{D}-\tilde{A}+\tilde{R}\right) \xi,
\end{aligned}
$$

${ }^{\dagger}$ Research partially supported by a DGICYT grant no PB94-0750.C02-01 and by a grant from Gobierno Vasco PI95/95.

Glasgow Math. J. 40 (1998) 205-212. 
where $\Delta^{D}$, denotes the Laplacian relative to the normal connection $D$ in $N(M), \tilde{A}$ is the Simons operator [11], defined on $N(M)$ by

$$
<\tilde{A} \xi, \eta>=\operatorname{trace}\left(A_{\xi} \circ A_{\eta}\right), \quad \xi, \eta \in N(M),
$$

with $A$ denoting the Weingarten map, and

$$
\tilde{R} \xi=\sum_{i=1}^{p}\left(\bar{R}\left(E_{i}, \xi, E_{i}\right)^{\perp},\right.
$$

where $\perp$ denotes the normal component and $\tilde{R}$ is the Riemannian curvature of $\tilde{M}^{n}$. A crosssection $\xi \in N(M)$ is called a Jacobi field [11], if $J \xi=0$.

Suppose that $M^{p}$ is compact. In this case we define an inner product in $N(M)$ by

$$
(\xi, \eta)=\int_{M}<\xi, \eta>d v
$$

Then $J$ is self-adjoint with respect to (4). Moreover $J$ is a strongly elliptic operator and it has an indefinite sequence of distinct eigenvalues

$$
\operatorname{Spec}(M, J)=\left\{\mu_{1}<\mu_{2}, . .<\mu_{k}<\ldots \rightarrow \infty\right\} .
$$

Let $\Gamma_{k}=\left\{\xi \in N(M) / J \xi=\mu_{k} \xi\right\}$ be the eigenspace of $J$ associated with $\mu_{k}$. Then the dimension of $\Gamma_{k}$, that is the multiplicity of $\mu_{k}$, is finite. Finally we know that the decomposition $\sum_{k=1}^{\infty} \Gamma_{k}$ is orthogonal with respect to (4) and it is dense in $N(M)$ in the $L_{2}$-sense.

For each $\xi \in N(M)$, let $\xi_{k}$ be the projection of $\xi$ onto $\Gamma_{k}$. Then one has the following spectral decomposition

$$
\xi=\sum_{k=1}^{\infty} \xi_{k}, \quad \text { (in the } L_{2} \text {-sense) }
$$

3. The J-type of spherical immersions. Assume that $x: M^{p} \rightarrow S^{n}(1) \subset E^{n+1}$ is an isometric immersion of a compact Riemannian manifold in the unit $n$-dimensional sphere, $S^{n}(1)$, centered at the origin of the Euclidean space $E^{n+1}$. The immersion $\left(M^{p}, x\right)$ is said to be of Chen-type $k$ [4], if the position vector $x$ can be decomposed in the following way:

$$
x=v+\sum_{i=1}^{k} x_{i_{i}}
$$

where $v \in E^{n+1}$ is a constant vector and $\Delta x_{t_{i}}=\lambda_{t_{i}} x_{t_{i}}, \Delta$ being the Laplacian of $M^{p}$ and $\lambda_{t_{i}} \in R$. Moreover, if the centre of mass of $\left(M^{p}, x\right)$ coincides with that of $S^{n}(1)$, then the immersion is called mass-symmetric [4].

For an isometric immersion $x: M^{p} \rightarrow S^{n}(1) \subset E^{n+1}$ the Jacobi operator $J$ on the normal bundle $N(M)$ is nothing but

$$
J=\Delta^{D}-\tilde{A}
$$


On the other hand, since $x \in N(M)$ it admits a spectral decomposition with respect to $J$ as in (5), given by

$$
x=\sum_{i=1}^{\infty} x_{i}, \quad x_{i} \in \Gamma_{i} .
$$

We say that $\left(M^{p}, x\right)$ is of $J$-type $k$ if only $\mathrm{k}$ non-zero members appear in (7). In particular, it is of $J$-type 2 if

$$
x=x_{s}+x_{r}
$$

with $x_{s}, x_{r} \in N(M)$ and $J x_{s}=\mu_{s} x_{s}, J x_{r}=\mu_{r} x_{r}, \mu_{s}, \mu_{r} \in R$. First we prove the following result.

Proposition 1. Let $x: M^{p} \rightarrow S^{n}(1) \subset E^{n+1}$ be an isometric immersion of a Riemannian manifold in the unit sphere. Then

$$
J x=-\Delta x=p H
$$

$H$ being the mean curvature vector of $M^{p}$ in $E^{n+1}$. In particular,

(i) $J x=\mu x, \mu \in R$, if and only if, $\left(M^{p}, x\right)$ is minimal in $S^{n}(1)$. Moreover, in this case, $-p \in \operatorname{Spec}\left(M^{p}, J\right)$.

(ii) $\mu_{1} \leq-p$. Equality holds, if and only if, $\left(M^{p}, x\right)$ is minimal in $S^{n}(1)$.

Proof. From (6) we get

$$
J x=\Delta^{D} x-\tilde{A} x=-\tilde{A} x
$$

Now, choose an orthonormal basis $\left\{\eta_{p+1}, \ldots, \eta_{n},-x\right\}$ normal to $M^{p}$ in $E^{n+1}$. Since each element of $\left\{\eta_{p+1}, \ldots, \eta_{n}\right\}$ is perpendicular to $M^{p}$ in $S^{n}(1)$, we have $A_{\eta_{i}}^{\prime}=A_{\eta i}$, where $A^{\prime}$ and $A$ are the Weingarten maps of $M^{p}$ in $S^{n}(1)$ and in $E^{n+1}$ respectively. Therefore we have from (10) and (2),

$$
\begin{aligned}
J x= & -\left\{\sum_{r=p+1}^{n} \operatorname{trace}\left(A_{x} \circ A_{\eta r}\right) \eta_{r}\right\}-\operatorname{trace}\left(A_{x} \circ A_{-x}\right)(-x) \\
& =\left\{\sum_{r=p+1}^{n} \operatorname{trace} A_{\eta r}^{\prime} \eta_{r}\right\}-p x=p H^{\prime}-p x=p H,
\end{aligned}
$$

where $H^{\prime}$ denotes the mean curvature vector of $M^{p}$ in $S^{n}(1)$. Thus (11) and the Beltrami equation, $\Delta x=-p H$ end the proof of (9). Part (i) is precisely Takahashis's theorem, (see [4], p. 136). To prove part (ii) one can apply $J$ to (7) and use (9) to obtain

$$
p H=\sum_{k=1}^{\infty} \mu_{k} x_{k} .
$$


But for compact submanifolds of $E^{m}$ we have $\int_{M}\{1+<x, H>\} d v=0$. Then the scalar product with respect to (4) of (12) with $x$ gives

$$
-p \cdot \operatorname{Vol}(M)=\sum_{k=1}^{\infty} \mu_{k}\left\|x_{k}\right\|^{2}
$$

Thus, there must be a negative eigenvalue $\mu_{j}$. Then

$$
p \cdot \operatorname{Vol}(M)=\sum_{k=1}^{\infty}\left(-\mu_{k}\right)\left\|x_{k}\right\|^{2} \leq-\mu_{1} \sum_{k=1}^{\infty}\left\|x_{k}\right\|^{2}=-\mu_{1} \cdot \operatorname{Vol}(M)
$$

Hence $\mu_{1} \leq-p$. Equality in (13) holds if and only if $\mu_{1}=\mu_{k} \forall k$.

This shows the equivalence of spherical submanifolds of Chen-type 1 with those of $J$-type 1 . Now, we study the spherical immersions whose immersions are constructed with two eigenvectors of $J$. Given $x: M^{p} \rightarrow S^{p+1}(1) \subset E^{p+2}$ an isometric immersion of a compact hypersurface in $S^{p+1}(1)$, we denote by $H, \alpha, \sigma$ the mean curvature vector, mean curvature function and scalar curvature of the immersion in $E^{p+2}$ respectively and by $H^{\prime}, \alpha^{\prime}, \sigma^{\prime}$ the corresponding elements in $S^{p+1}(1)$. First, we establish the following result.

Lemma 2. Let $x: M^{p} \rightarrow S^{p+1}(1) \subset E^{p+2}$ be an isometric immersion of a compact hypersurface in $S^{p+1}(1)$. Then $\left(M^{p}, x\right)$ has constant mean curvature if an only if $J H=-\Delta H$.

Proof. We shall use the following formula which was proved in [2] (see also [18])

$$
\Delta H=J H+2 \tilde{A} H+(\Delta H)^{T},
$$

where ()$^{T}$ denotes the tangential part to $M^{p}$. Thus combining (14) and (6) we have

$$
J H=-\Delta H+2 \Delta^{D} H+(\Delta H)^{T},
$$

where $(\Delta H)^{T}$ is given by [3]

$$
(\Delta H)^{T}=\frac{p}{2} \nabla \alpha^{2}+2 \operatorname{trace} A_{D H}
$$

Then it is enough to observe that $(\Delta H)^{T}$ is tangential, $\Delta{ }^{D} H$ is normal and that they are both zero if and only if $\alpha$ is constant.

The following Proposition relates the $J$-type to the Chen-type for spherical hypersurfaces

Proposition 3. Let $x: M^{p} \rightarrow S^{p+1}(1) \subset E^{p+2}$ be an isometric immersion of a compact hypersurface in $S^{p+1}(1)$. Then $\left(M^{p}, x\right)$ is of J-type 2 if and only if it is of Chen-type 2 or it is a small hypersphere.

Proof. We shall need the following Chen's formula (See Lemma 4.2 of [4]).

$$
\Delta H=\Delta^{D} H+(\Delta H)^{T}+\|\sigma\|^{2} H^{\prime}-p \alpha^{2} x .
$$


Assume first that $\left(M^{p}, x\right)$ is of $J$-type 2. Then $x=x_{r}+x_{s}$ with $J x_{s}=\mu_{s} x_{s}, J x_{r}=\mu_{r} x_{r}$. Hence $J x=\mu_{r} x_{r}+\mu_{s} x_{s}$ and $J^{2} x=\mu_{r}^{2} x_{r}+\mu_{s}^{2} x_{s}$. From these two equations we have

$$
J^{2} x=b J x+c p x
$$

where $b=\mu_{r}+\mu_{s}$ and $c=-\frac{\mu_{r} \mu_{s}}{p}$. Using (9) in (18) one has

$$
J H=b H+c x
$$

Combining (15), (17) and (19), we find that $M^{p}$ must have constant mean curvature and constant length of the second fundamental form given by

$$
\alpha^{2}=\frac{c-b}{p} ;\|\sigma\|^{2}=-b .
$$

Then, by using (9), Lemma 2 and (18), we have

$$
\Delta^{2} x-b \Delta x+c x=0
$$

and so it must be of Chen-type 2 if $c \neq 0$ and a small hypersphere if $c=0$, [4].

Conversely, assume that $M^{p}$ is a small hypersphere with centre $x_{o}$ (which is perpendicular to $\left.M^{p}\right)$; then it is known that $\Delta\left(x-x_{0}\right)=\lambda\left(x-x_{0}\right)$, and so, by using Proposition 1 , we have

$$
J\left(x-x_{o}\right)=J x-J x_{0}=-\Delta\left(x-x_{o}\right)=-\Delta x=J x .
$$

Thus $x=\left(x-x_{0}\right)+x_{0}$ with $J\left(x-x_{0}\right)=-\lambda\left(x-x_{0}\right)$ and $J x_{0}=0$. Therefore it is of $J$-type 2 . Now we suppose that $\left(M^{p}, x\right)$ is of Chen-type 2 . Then it is mass-symmetric and has constant mean and scalar curvatures [1], [8]. Thus we have by [4]

$$
\Delta^{2} x+b_{1} \Delta x+c_{1} x=0
$$

Since $\alpha$ is constant, we use Lemma 2 and (9) in (21) to obtain

$$
J^{2} x-b_{1} J x+c_{1} x=0
$$

Since the position vector $x$ lies in $N(M)$, we have according to (7)

$$
x=\sum_{k=1}^{\infty} x_{k}
$$

with $J x_{k}=\mu_{k} x_{k}$. But (22) and (23) give

$$
\sum_{k=1}^{\infty}\left(\mu_{k}^{2}-b_{1} \mu_{k}+c_{1}\right) x_{k}=0 .
$$


Scalar multiplication with $x_{k}$ (with respect to (4)) gives

$$
\left(\mu_{k}^{2}-b_{1} \mu_{k}+c_{1}\right)\left\|x_{k}\right\|^{2}=0,
$$

which proves that all the $x_{k}$ must be zero except at most two of them. But if only one were different from zero, then Proposition 1 would show that $\left(M^{p}, x\right)$ is of Chen-type 1 , and this is a contradiction. Hence, there must be exactly two $x_{k}$ different of zero and $\left(M^{p}, x\right)$ is of $J$-type 2.

Remark 1. It is not difficult to see that if $x: M^{p} \rightarrow S^{p+1}(1)$ is an immersion of Chentype 2 and $x=x_{i}+x_{q}$ is the spectral decomposition with respect to $\Delta$ with eigenvalues $\lambda_{t}$ and $\lambda_{q}$, then $x=x_{t}+x_{q}$ is also the spectral decomposition with respect to $J$ with eigenvalues $\mu_{t}=-\lambda_{t}, \mu_{q}=-\lambda_{q}$. Since $M^{p}$ is compact, the eigenvalues of $J$ in the $J$-type 2 decomposition of $x$ must be non-positive.

The following two results are a reformulation, using the above Proposition, of known facts on Chen-type 2 immersions [6].

COROLlaRy 4. Let $x: M^{p} \rightarrow S^{p+1}(1)$ be an isometric immersion of a compact Riemannian manifold in the unit sphere which is not a small hypersphere. Then $\left(M^{p}, x\right)$ is of J-type 2 if and only if it has constant mean and scalar curvatures.

COROllaRY 5. Let $x: M^{2} \rightarrow S^{3}(1)$ be an isometric immersion of a closed surface in the unit 3-sphere. Then $\left(M^{2}, x\right)$ is of J-type 2 if and only if $M^{2}$ is the standard immersion of the flat torus $S^{1}(a) \times S^{1}(b)$ with $a \neq b$.

4. An application. Now let $J^{\prime}$ be the Jacobi operator defined on the normal bundle of $M^{p}$ in $S^{p+1}(1)$. If $\eta^{\prime}$ is a normal vector field to $M^{p}$ in $S^{p+1}(1)$, then

$$
J^{\prime} \eta^{\prime}=\left(\Delta^{D^{\prime}}-\tilde{A}^{\prime}+\tilde{R}^{\prime}\right) \eta^{\prime}
$$

where $D^{D^{\prime}}, \tilde{A}^{\prime}, \tilde{R}^{\prime}$ are the operators defined through (1) to (3) relative to the normal bundle of $M^{p}$ in $S^{p+1}(1)$.

By a straightforward computation, the following relation is obtained

$$
J H^{\prime}=J^{\prime} H^{\prime}+p H^{\prime}+p \alpha^{\prime 2} x
$$

$H^{\prime}$ being the mean curvature vector of $M^{p}$ in $S^{p+1}$. Therefore we have

Lemma 6. Let $x: M^{p} \rightarrow S^{p+1}(1) \subset E^{p+2}$ be an isometric immersion of a compact Riemannian manifold in the unit sphere. Then the mean curvature vector field $H^{\prime}$ in $M^{p}$ in $S^{p+1}$, is a Jacobi vector field for the Jacobi operator of the normal bundle of $M^{p}$ in $E^{p+2}$, if and only if $M^{p}$ is minimal in $S^{p+1}(1)$. 
As an application we obtain the following result:

Proposition 7. Let $M^{p}$ be a closed hypersurface of the unit hypersphere $S^{p+1}(1)$. Suppose that it has constant mean and scalar curvatures and that $M^{p}$ and $S^{p}(1)$ are isospectral with respect to J, then $M^{p}=S^{p}(1)$.

Proof. Using Corollary 4, we see that $M^{p}$ is either a small hypersphere or of $J$-type 2. Isospectrality implies in the first case that $M^{p}=S^{p}(1)$. Assume then that it is of $J$-type 2 . Thus its position vector decomposes $x=x_{r}+x_{s}$ with $J x_{r}=\mu_{r} x_{r} ; J x_{s}=\mu_{s} x_{s}$ and we assume that $\mu_{r} \leq \mu_{s} \leq 0$. Then, from (20), one has

$$
\alpha^{\prime 2}=-\frac{b}{p}+\frac{c}{p}-1 ;\left\|\sigma^{\prime}\right\|^{2}=-p-b
$$

with $b=\mu_{r}+\mu_{s}$ and $c=-\frac{\mu_{r} \mu_{s}}{p}$, and $\alpha^{\prime}, \sigma^{\prime}$ being the mean curvature function and second fundamental form of $M^{p}$ in $S^{p+1}(1)$ respectively. By using the asymptotic expansion of the heat kernel of $J$ [7], we get in our case that

$$
\frac{7}{6}\left\|\sigma^{\prime}\right\|^{2}-\frac{1}{6} \alpha^{\prime 2}
$$

is a spectral invariant. In particular, since $S^{p}(1)$ is totally geodesic, we have from (28)

$$
b=-p-\frac{c}{7 p-1}
$$

but $-c \geq 0$ gives

$$
b \geq-p
$$

Now, we wish to prove that $\mu_{r} \leq-p$. Using Proposition 1,

$$
(x, J x)=p \cdot(x, H)=p \int_{M}<x, H>=\mu_{r}\left\|x_{r}\right\|^{2}+\mu_{s}\left\|x_{s}\right\|^{2},
$$

where \|\| denotes the norm with respect to the inner product (4). Since $\int_{M}(1+<x, H>)=0$, one has from (31)

$$
p \cdot \operatorname{Vol}(M)=-\mu_{r}\left\|x_{r}\right\|^{2}-\mu_{s}\left\|x_{s}\right\|^{2} .
$$

Therefore, combining (32) and $\left\|x_{r}\right\|^{2}+\left\|x_{s}\right\|^{2}=\|x\|^{2}=\operatorname{Vol}(M)$, one obtains $\mu_{r} \leq-p \leq$ $\mu_{s} \leq 0$. Hence

$$
b=\mu_{r}+\mu_{s} \leq-p
$$

From (30) and (33) we have

$$
b=-p
$$

which together with (29) gives

$$
c=0
$$


Consequently $\mu_{r}=-p$ and $\mu_{s}=0$ which means that $x_{s}$ is a Jacobi field for $J$. By Proposition 1, $H=-x_{r}$ and therefore, since $H=H^{\prime}-x$, we have $H^{\prime}=-x_{s}$. Thus $H^{\prime}$ is a Jacobi field for $J$ and Lemma 6 gives $H^{\prime}=0$ (observe also that $H^{\prime}=0$ implies $x=x_{r}$ so that by Proposition 1, $M^{p}$ would be minimal.) Then the result follows from Corollary 3.3 of [7].

COROLlaRY 8. No spherical hypersurface $M^{p} \subset S^{p+1}(1)$ of Chen-type 2 is isospectral (with respect to J) with $S^{p}(1)$.

\section{REFERENCES}

1. M. Barros, A. Ferrández and P. Lucas, Spherical 2-type hypersurfaces. Pulicaciones del Departamento de Matemáticas. Univ. de Murcia. No 1 (1991).

2. M. Barros and O. J. Garay, Euclidean submanifolds with Jacobi mean curvature vector field, to appear in J. Geometry.

3. B.-Y. Chen, 2-type submanifolds and their applications, Chinese J. Math. 14 (1986), 1-14. 1984).

4. B.-Y. Chen, Total mean curvature and submanifolds of finite type, (World Scientific, Singapore,

5. B.-Y. Chen, Some open problems and conjectures on submanifolds of finite type, Soochow $J$. Math. 17 (1991), 169-188.

6. B.-Y. Chen, A report on submanifolds of Finite Type, Soochow J. Math. 22 (1996), 117-337. 185-189.

7. H. Donnelly, Spectral invariants of the second variation operator, Illinois J. Math. 21 (1977),

8. T. Hasanis and T. Vlachos, Spherical 2-type Hypersurfaces, J. Geometry 40 (1991), 82-94.

9. T. Hasegawa, Spectral geometry of closed minimal submanifolds in a space form real or complex, Kodai Math. J. 3 (1980), 224-252.

10. S.-J. Li and C.-S. Houh, Generalized Chen submanifolds, to appear in J. Geometry. (special issue dedicated to Professor A. Barlotti).

11. J. Simons, Minimal varieties in Riemannian manifolds, Ann. of Math. 88 (1968), 62-105.

J. Arroyo and O. J. Garay

Departamento de Matemáticas

UNIVERSIDAD DEL PAÍS VASCO/EHU

Apto 644. 48080 Bilbao

SPAIN

\author{
M. Barros \\ Departamento de Geometría y Topología \\ UNIVERSIDAD DE GRANADA \\ 18071 Granada \\ SPAIN
}

